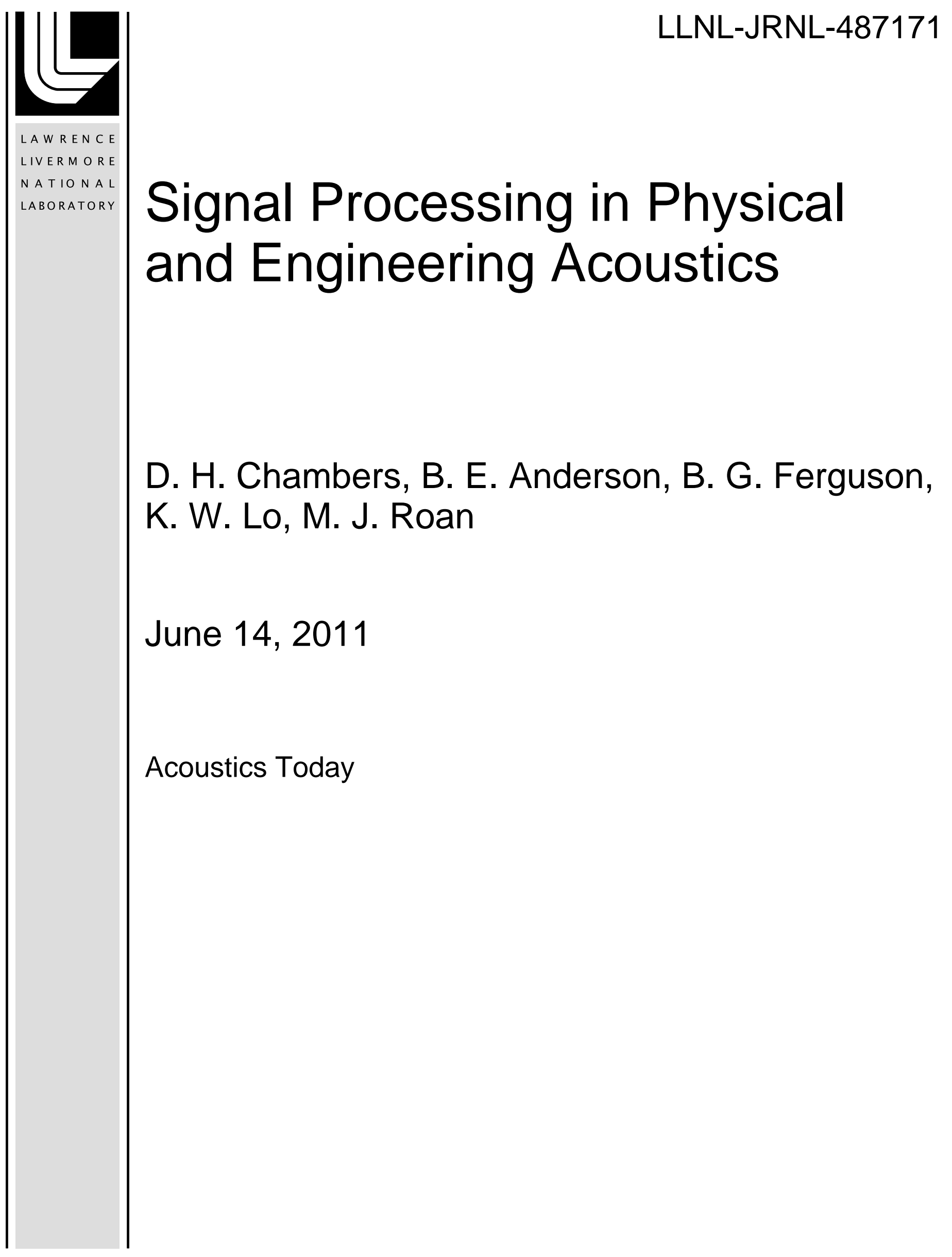


This document was prepared as an account of work sponsored by an agency of the United States government. Neither the United States government nor Lawrence Livermore National Security, LLC, nor any of their employees makes any warranty, expressed or implied, or assumes any legal liability or responsibility for the accuracy, completeness, or usefulness of any information, apparatus, product, or process disclosed, or represents that its use would not infringe privately owned rights. Reference herein to any specific commercial product, process, or service by trade name, trademark, manufacturer, or otherwise does not necessarily constitute or imply its endorsement, recommendation, or favoring by the United States government or Lawrence Livermore National Security, LLC. The views and opinions of authors expressed herein do not necessarily state or reflect those of the United States government or Lawrence Livermore National Security, LLC, and shall not be used for advertising or product endorsement purposes. 
Signal processing is used extensively in physical and engineering acoustics, with applications in nondestructive evaluation, machine and structural monitoring, tracking and localization, and elsewhere.

\section{Signal Processing in Physical and Engineering Acoustics}

\section{David H. Chambers ${ }^{a}$, Brian E. Anderson ${ }^{b}$, Brian G. Ferguson ${ }^{c}$, Kam W. Lo ${ }^{c}$, Michael J. Roan ${ }^{\mathrm{d}}$}

${ }^{a}$ Lawrence Livermore National Laboratory, PO Box 808, L-154, Livermore, CA, 94551

${ }^{\mathrm{b}}$ Acoustics Research Group, Department of Physics and Astronomy, Brigham Young University Provo, UT 84602

${ }^{\mathrm{c}}$ Maritime Operations Division - Sydney, Defence Science and Technology Organisation, PO Box 44 Pyrmont 2009 NSW Australia

${ }^{\mathrm{d}}$ Department of Mechanical Engineering, Virginia Polytechnic Institute and State University, 100S Randolph Hall, Blacksburg, VA 24061

\section{Introduction}

Signal processing is used extensively in physical and engineering acoustics, with applications in nondestructive evaluation, machine and structural monitoring, tracking and localization, and elsewhere. The goal of signal processing is to extract information from noisy and uncertain measurements. In this process we exploit both statistical analysis and properties of acoustic wave generation and propagation to separate extraneous components of the measurements from the signal of interest. Due to the breadth of applications and methods, we have decided not to attempt a survey of signal processing physical and engineering acoustics. Rather we present three examples of signal processing that illustrate different methods and approaches to the problem of extracting information from measurements. The first example uses the symmetry of reciprocal wave propagation and timing of reflections detect flaws (cracks) in plates. This is an illustration of a signal processing exploiting a principle associated with physical acoustics. The second example uses a sophisticated statistical approach to determine the condition of gears in a gearbox from accelerometer measurements. Machine condition monitoring is a large area of engineering acoustics motivated by both cost and safety. The final example shows how combining information from different sound sources improves the ability to locate the origin of a bullet fired from a firearm.

\section{Time Reversal ${ }^{1}$}

Time reversal (TR) is a method of locating and characterizing sources and to intentionally focus energy at a selected location in space. ${ }^{2-3}$ The original time reversal 
experiments were conducted by Parvulescu and Clay in the early 1960s to demonstrate the reproducibility of signal transmissions in the ocean. ${ }^{4}$ It has found application in SONAR, ${ }^{5}$ communications, ${ }^{6-7}$ medical ultrasound, ${ }^{8-9}$ nondestructive evaluation, ${ }^{10-11}$ and seismic imaging ${ }^{12-}$

${ }^{13}$ (see selected references for recent work in these areas). The application of TR to nondestructive evaluation allows localization of cracks, ${ }^{10}$ which are nonlinear scatterers, and linear passive scatterers. ${ }^{11}$ It will likely soon be shown that TR can be used to locate acoustic emission events, as work is currently underway to show this.

To illustrate the application of TR to nondestructive evaluation, we will describe a basic TR experiment. During the forward propagation step, a source signal is broadcast from location $A$ in a bounded sample. A reversible transducer at location $B$ collects the directly propagated signal from $A$ and reflections of the source signal from the various possible reflected paths between $A$ and $B$. The signal recorded at $B$ is then reversed in time and during the backward propagation step is broadcast from the reversible transducer at $B$. This second broadcast signal traverses the propagation paths traversed during the forward propagation step. The energy broadcast along each respective path is timed such that they will simultaneously arrive at $A$.

Reverse time migration (RTM) is a variant of TR commonly used in seismology applications to image scatterers of interest in the ground. Anderson et al. recently demonstrated that RTM may be fully implemented experimentally in 2-D laboratory samples to image scatterers on an inaccessible side of a plate and places where these scatterers may be partially delaminated. ${ }^{11}$ To image scatterers using RTM, the aforementioned TR experiment is carried out as normal, however during both the forward and backward propagation steps the vibration of the wave field at various points within a region of interest (ROI) must be sensed, with a scanning laser vibrometer for example. RTM imaging correlates the arrivals of energy at specific times at a particular scatterer during the forward propagation with corresponding arrivals of energy at analogous times at the same scatterer during the broadcast and convergence of energy of the backward propagation (after these last set of data have been reversed in time). To the degree that the energy broadcast during the backward propagation step retraces the forward propagation paths, RTM allows imaging of passive scatterers.

Figure 1a illustrates the forward propagation of a RTM experiment conducted in free space with a source at $A$, a reversible transducer at $B$ and a scatterer at $C$. The forward signal is emitted from $A$ after 7 time units. This forward signal is then directly received at $B$ at a time of 11 units and the reflection off of $C$ arrives at $B$ at a time of 12 units. The forward signal arrives at $C$ at a time of 10 units. The signal recorded at $B$ is now flipped in time and used as the source signal for the backward propagation depicted in Fig. 1b (we color the two emission signals from $B$ to aid visualization of this step). The red signal from $B$ directly travels to $A$ and arrives at a time of 6 units. The red signal from $B$ also reflects off of $C$ and arrives at $A$ at a time of 7 units. The blue signal from $B$ also directly travels to $A$ and arrives at a time of 7 units (constructively interfering with the reflected red arrival producing the purple recorded signal). The blue signal from $B$ also reflects off of $C$ and arrives at $A$ at a time of 8 units. The signal at $A$ is typical of a symmetric time reversal focus signal. ${ }^{13}$ The signal recorded at $C$ consists of the red signal delayed by 2 time units and the blue signal delayed by 2 units. Now, if the signal recorded at $C$ is reversed in time and compared to the signal recorded at $C$ during the forward propagation step, one will notice that the green arrival and the red arrival are synced in time. The traditional RTM image, $I$, is found through computing the cross correlation of the forward signal, $F$, at position $(x, y)$ with the backward signal, $B$, at $(x, y)$, after this later signal has been reversed in time,

$$
I_{x, y}=\mathfrak{J}^{-1}\left[\mathfrak{J}\left\{F_{x, y}(t)\right\} \mathfrak{J}^{*}\left\{B_{x, y}(-t)\right\}\right] \text {, }
$$


where $\mathfrak{I}$ represents a Fourier transform, * represents phase conjugation, and $-t$ represents a time reversal. The magnitude of the image $\left|I_{x, y}\right|$ is then typically displayed to locate scatterers. Anderson et al. found that this traditional imaging condition doesn't work well in a highly reverberant medium and instead used the following imaging condition with better results

$$
M_{x, y}=\mathfrak{J}^{-1}\left|\mathfrak{J}\left\{F_{x, y}(t)\right\} \mathfrak{J}^{*}\left\{B_{x, y}(-t)\right\}\right| \text {. }
$$

The experiments conducted by Anderson et al. found that scatterers of a high impedance relative to the sample impedance showed up as minima in the $M_{x, y}$ image. In another experiment at Los Alamos National Laboratory, the question of whether $M_{x, y}$ could distinguish between low and high density scatterers was investigated ${ }^{15}$. This experiment utilizes a nearly semicircular aluminum plate of dimensions 6.54x179x396 mm (pictured in Fig. 2a). The characters "LANL" are milled out of the plate at a depth of $3.23 \mathrm{~mm}$ and a width of $10 \mathrm{~mm}$. The characters "EES-11" are cut out of a $2.64 \mathrm{~mm}$ thick steel plate with a width of $10 \mathrm{~mm}$ and glued onto the plate. The removal of plate material for the "LANL" characters should present an incident wave with a low impedance scatterer, while the addition of material for the "EES-11" characters should present a high impedance scatterer. In this experiment a single transducer (labeled as $\mathrm{S}$ in Fig. 2a and is slightly not in view for the photograph) is used as the source with a single receiver transducer (labeled as $\mathrm{R}$ in Fig. 2a). A scanning laser vibrometer scans the forward and backward propagation wave fields on the other side of the plate. The ROI in this experiment includes all of the characters with approximately a $1 \mathrm{~mm}$ spacing between scan points. The image $M_{x, y}$ of the characters is displayed in Fig. 2b. The characters "LANL" are clearly visible as maxima of $M_{x, y}$. The characters "EES-11" are not quite as visible. It is expected that these would show up as minima just as the high impedance scatterers used in the work of Anderson et al. The reason for the poorly visible "EES-11" characters may be due to the thickness of the plate relative to the wavelength, resulting in a smaller impedance contrast from the "EES-11" letters to the aluminum plate than the impedance contrast in the Anderson et al. experiments.

Thus, RTM imaging for nondestructive evaluation of bounded plate samples may be used to image passive scatterers and locations of disbonding of those scatterers. This procedure may be carried out to image scatterers on the opposite side of a thin plate if that side is inaccessible. It may also prove to distinguish between high and low impedance scatterers. The work of Anderson et al. showed that high impedance scatterers showed up as minima in a RTM image and the work presented here shows that low impedance scatterers show up as maxima, but further work needs to be done to determine why the high impedance scatterers considered here did not show up as minima. 
(a)

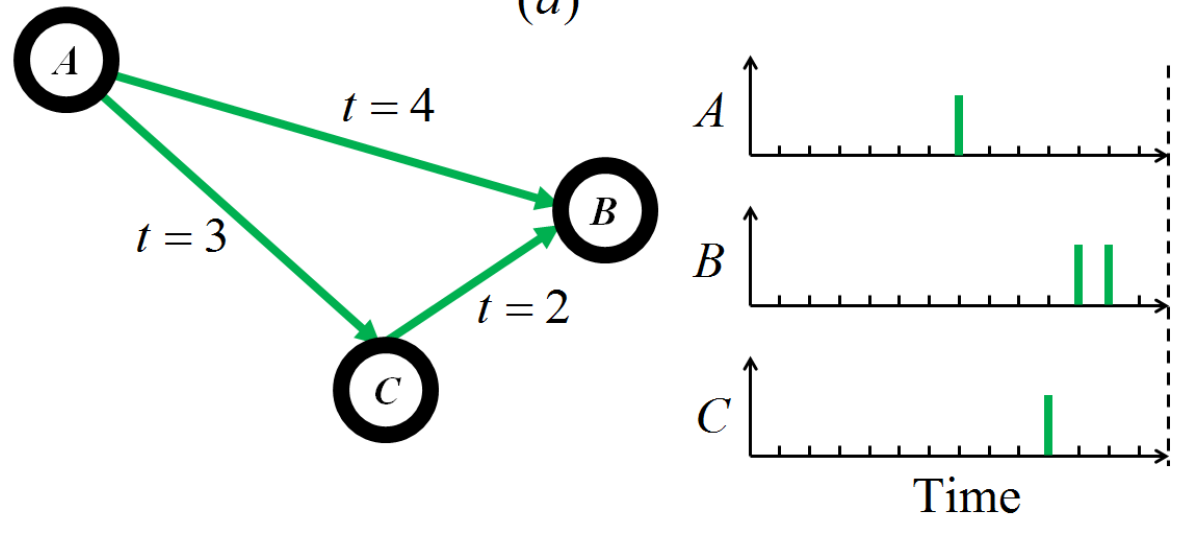

(b)
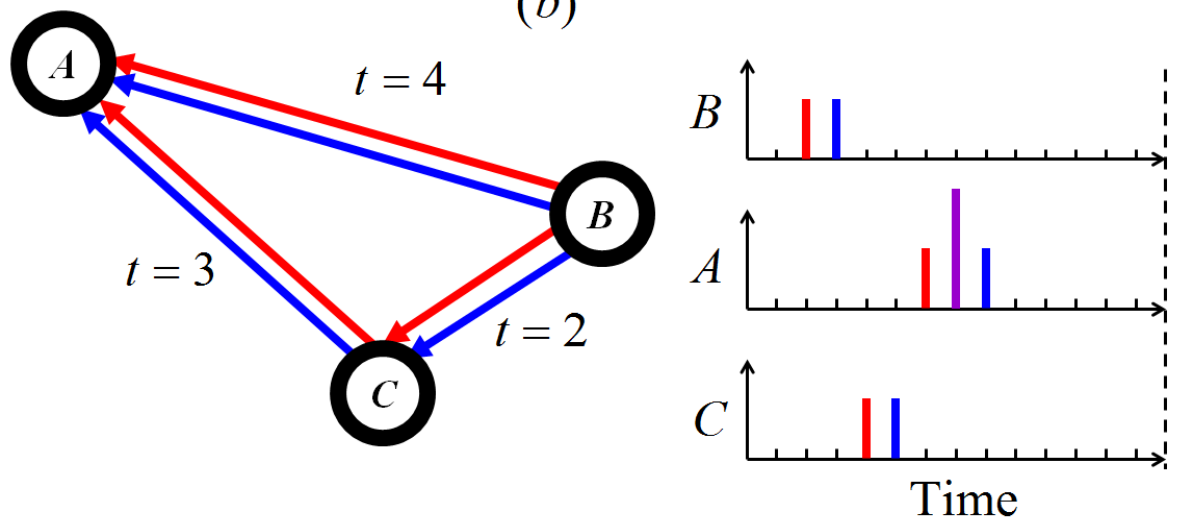

Figure 1. Illustration of the reverse time migration process in a free space with a scatterer at location $C$. The propagation times for each path are included for the reader's reference. (a) Forward propagation step. (b) Backward propagation step. 


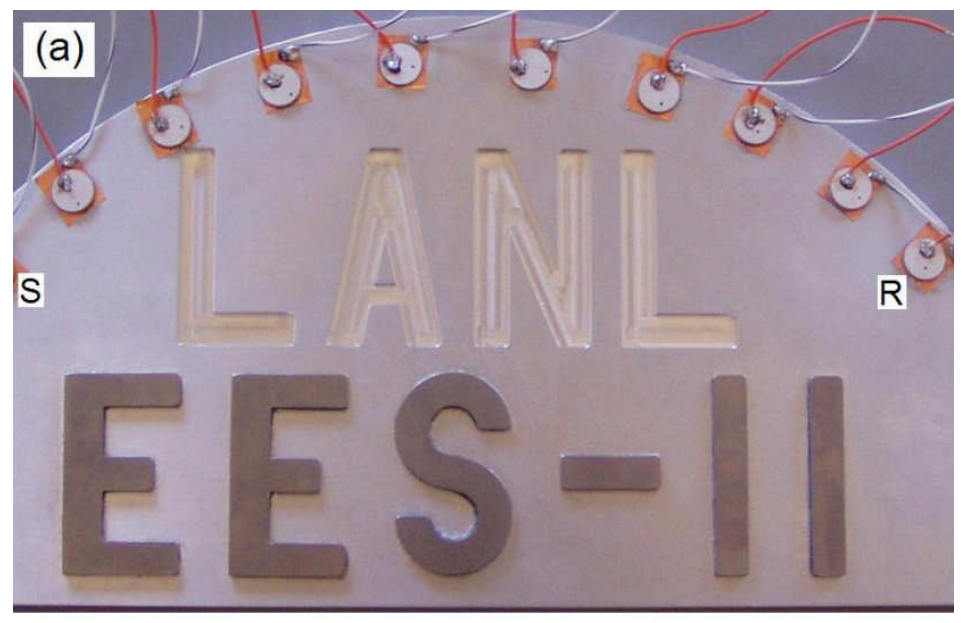

(b)

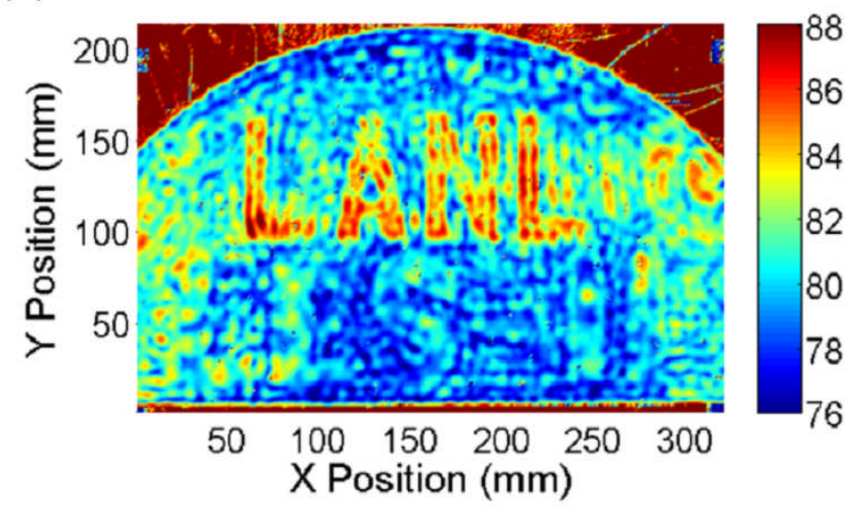

Figure 2. (a) Photograph of a semicircular aluminum plate which has the letters "LANL" milled out of it and steel letters "EES-11" glued onto it ${ }^{15}$. S and R denote the source and receiver transducers named according to the forward propagation usage. (b) RTM image of the other side of this plate (mirror image is displayed). Amplitude is in $\mathrm{dB}$ units with an arbitrary reference. 


\section{Independent Component Analysis Applied to Non-Invasive Gear Health Monitoring ${ }^{16}$}

The non-invasive monitoring of the health of gearboxes has been a very active area of research over the past two decades. The capability to predict gear failures from accelerometerbased measurements of the gear meshing vibration signal is of great import ance in industries such as the aerospace industry (e.g. helicopter gearboxes). If one can predict failure, then gears can be swapped during regular scheduled maintenance. This prevents accidents and saves money due to unscheduled down time. The main concept in gear health monitoring is that the meshing of the gear and pinion (Figure 3) gives rise to a vibration signal that propagates to the gearbox case where it is measured by accelerometers. The challenge is that, in real systems, many vibration signals arise due to vehicle motion, shaft imbalances, mode shape vibrations of the gearbox case, etc. All of these are also present at the measurement points necessitating the development of signal processing approaches that can isolate and analyze the gear me sh signal. Past condition monitoring techniques of gearboxes have used many different signal processing approaches such as synchronous time series averaging ${ }^{17}$, amplitude and phase demodulation ${ }^{18}$, time-frequency distribution ${ }^{19}$ and wavelet analysis ${ }^{20}$. The use of statistical signal processing approaches have also taken a hold in gear tooth failure detection ${ }^{21}$. Non-linear adaptive algorithms for independent component analysis (ICA) have been shown to separate unknown, statistically independent sources that have been mixed in dynamic systems. This example illustrates the application of an information maximization based blind source separation algorithm (a type of independent component analysis (ICA)) to the prediction of gear failures. It is shown that ICA can be used to detect impulsive and random changes in the gear vibration data.

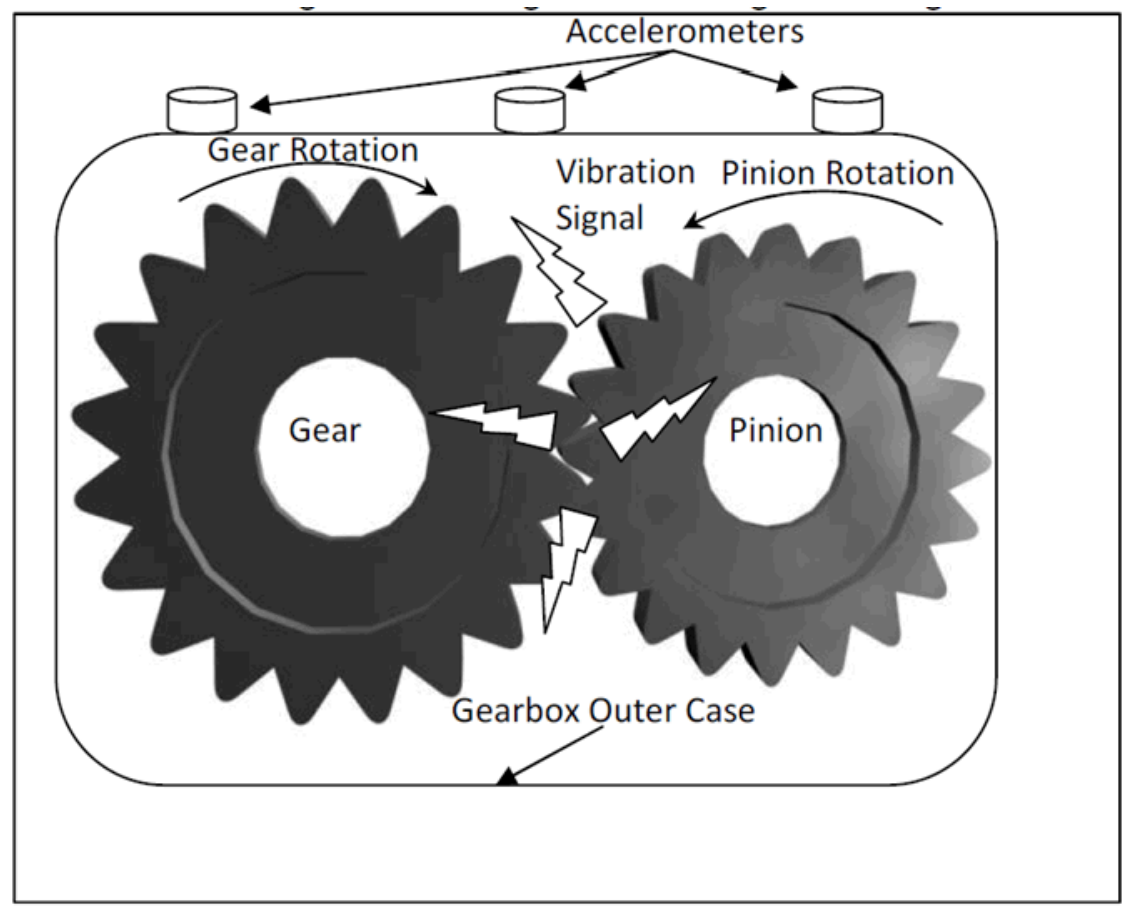

Figure 3. Simple spur gear and pinion setup

In typical gearbox setups, the pinion drives the gear through rotational motion where force is exerted on the gear teeth where the pinion teeth come into contact with the gear teeth. A common failure of the gear teeth occurs when a crack occurs at the base of the tooth due to material fatigue (Figure 4). 


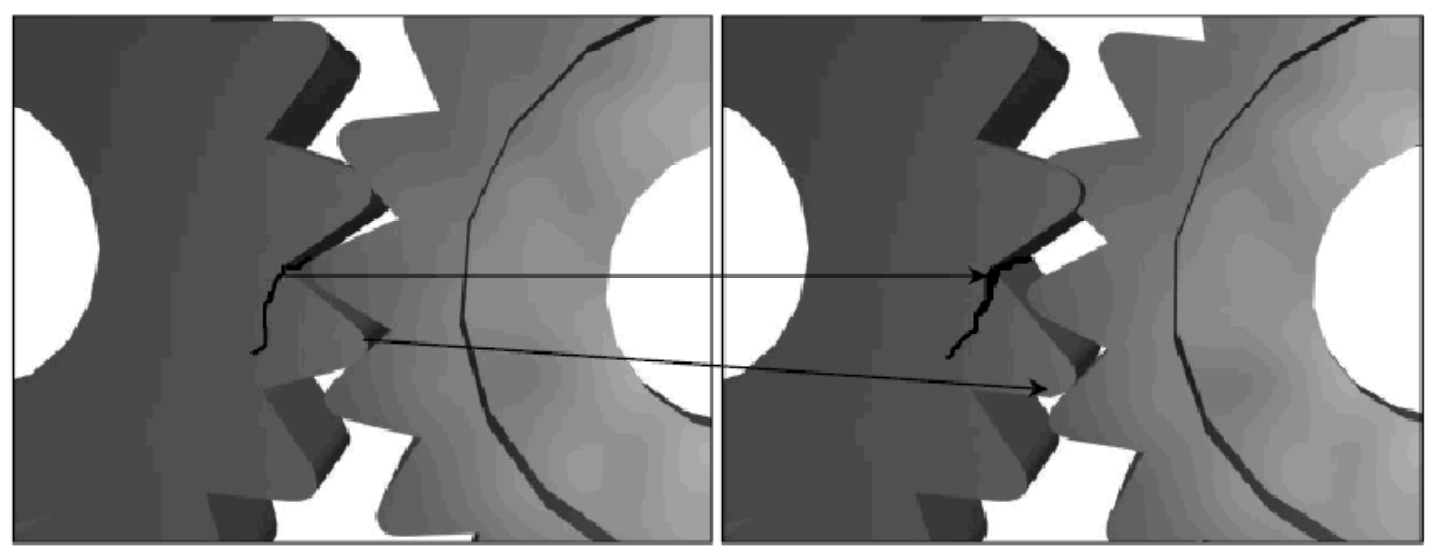

Figure 4. Crack formation and growth at the base of a gear tooth

The crack formation at the base of the tooth begins to cause the tooth to flex when the pinion exerts force on the tooth. Because the tooth flexes, there is a slight delay in the rotation of the gear causing a modulation of the meshing vibration signal. It is this modulation that fault monitoring algorithms attempt to detect before the tooth actually fails.

Having measured the signals at several locations on the gearbox surface, the next task is to separate the gear-pinion meshing signal and determine whether a change (or modulation) occurs during the gear-pinion contact period. One candidate algorithm for performing these tasks is the blind source separation algorithm (BSS).

Figure 5 shows a high level diagram of the BSS algorithm. The approach assumes that there are $r$ independent source signals that are linearly mixed by a mixing matrix $A$. This linear mixing is the mathematical model for signals from multiple sources arriving at each accelerometer. The signals $\mathrm{X}(\mathrm{t})$ are then measured at the accelerometer points and fed into the BSS part of the algorithm. BSS attempts to minimize the mutual information between accelerometer channels (i.e. measurements made at several points on the gearbox surface). This forces the independent sources $\hat{S}$ into separate channels. The weight matrix W, once learned, is ideally $\mathrm{A}^{-1}$. By observing changes in the trace of the weight matrix $\mathrm{W}$, one can observe points where the algorithm diverges (i.e. where there are abrupt statistical changes in the signals). By plotting the trace of $\mathrm{W}$ as a function of the gear tooth locations, the modulation of the meshing signal can be detected. Figure 6 gives a plot of the trace of $\mathrm{W}$ vs. gear tooth number for a healthy gear and for the same gear at a later time as it begins to fail. The point of the future failure is very visible in the lower plot. This approach provides the possibility to non-invasively monitor the health of gears or other types of rotating machinery such as bearings using measurements made at the gearbox (or machine) surface and can in theory separate out all of the unwanted signals that are present in the mixture of signals at the measurement points. This allows an analysis of the "clean" signatures alone. This is the reason that ICA/BSS algorithms have seen wide application from voice processing to the isolation of sounds in the human chest cavity. 


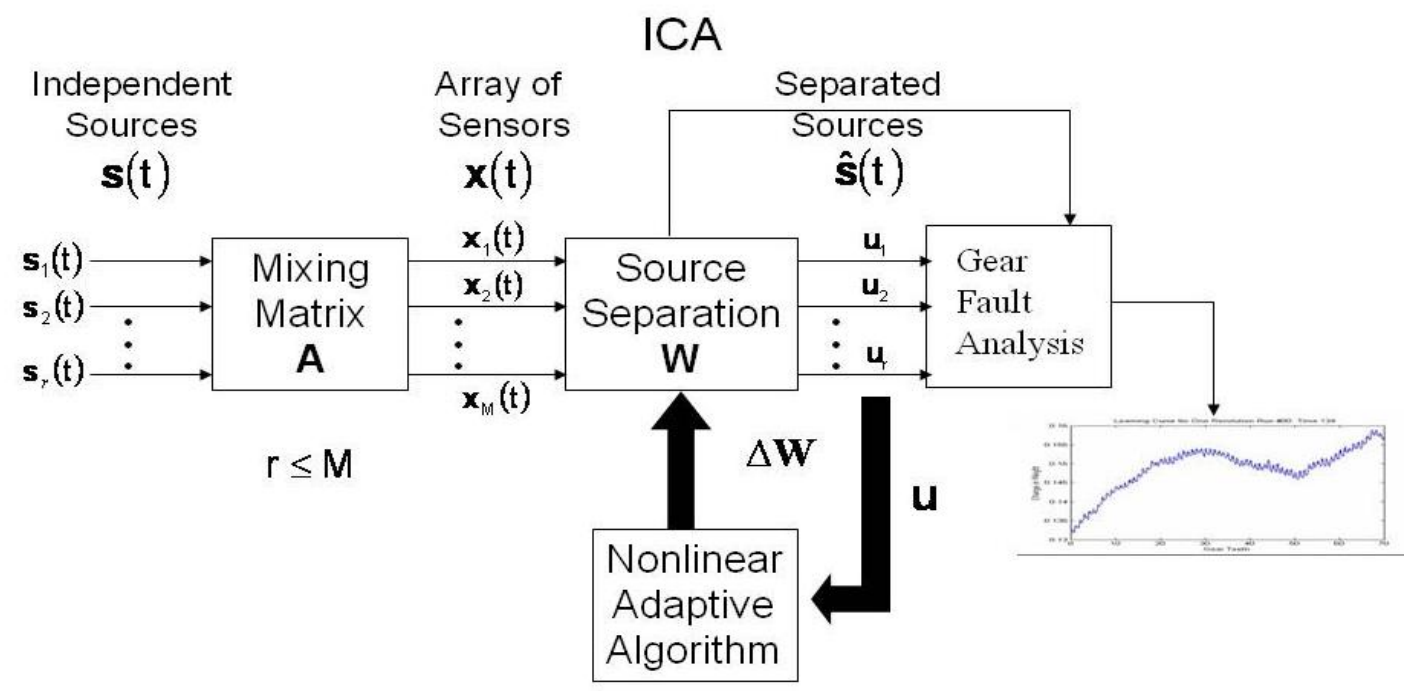

Figure 5. High Level BSS Algorithm
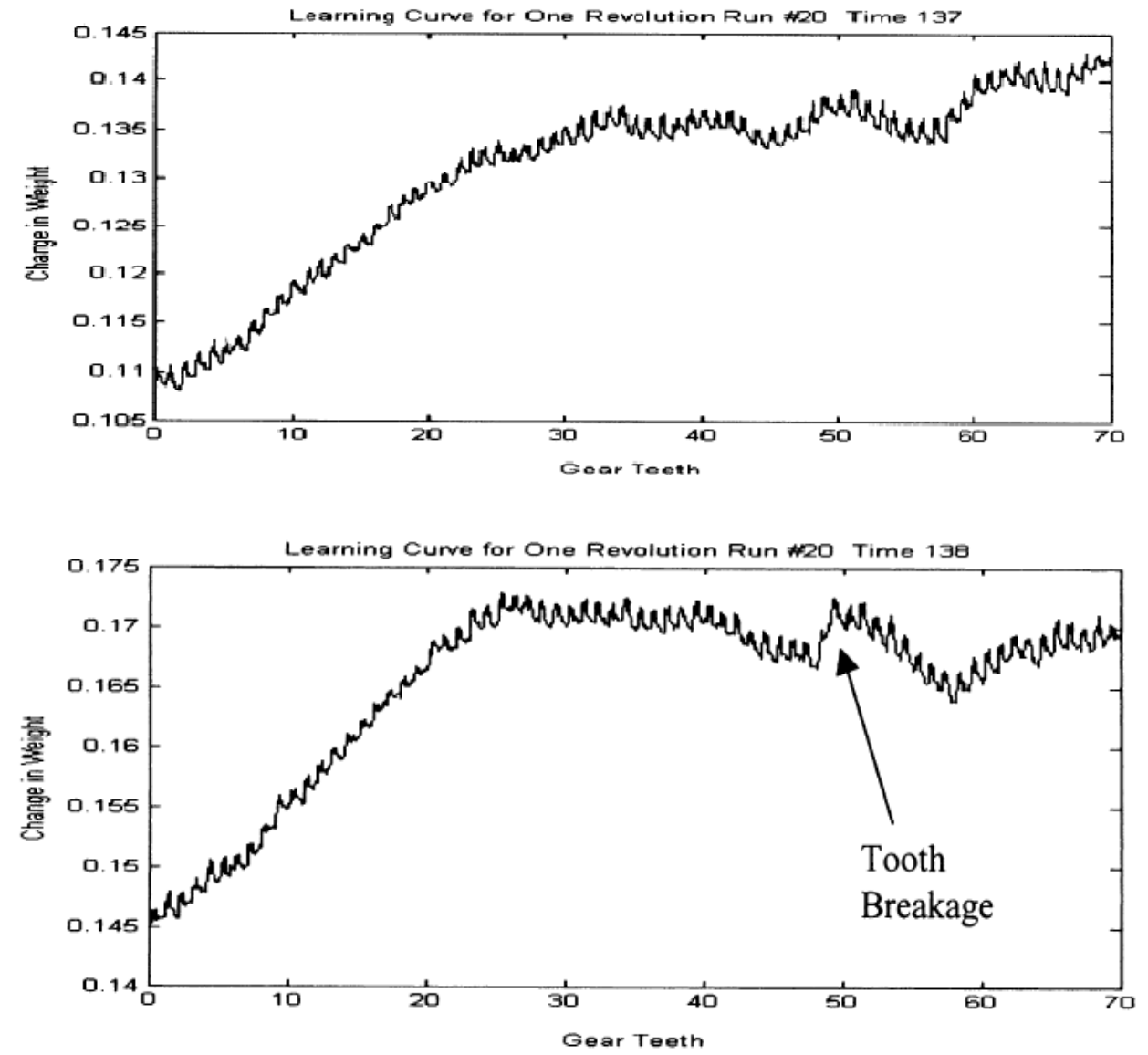

Figure 6. Result of BSS Processing for a healthy gear (top) and for a gear that is beginning to break (bottom) 


\section{Acoustical Signal Processing Methods for Localizing Gunshots ${ }^{22}$}

To an unalerted listener, the sound of gunfire represents an aural event in the soundscape that evokes a reaction of surprise tempered by curiosity. However, in engineering acoustics it represents a transient acoustic signal generated by the discharge of a firearm from which information can be extracted such as the location of the point of fire (localization) and the type of firearm (classification). The sound pulse generated by the discharge of a bullet from a firearm is referred to as the muzzle blast or in military parlance - the report. The acoustic energy propagates at the speed of sound travel in air and expands as a spherical wavefront (of constant phase) centered on the point of fire. Since the propagation of the sound through the atmosphere is omnidirectional, it can be heard from any direction, even behind the firer. If the listener is positioned forward (towards the front) of the firer and the bullet travels at supersonic speed, then a second transient signal, which is referred to as the (ballistic) shock wave is heard. To the listener, the origin of the shock wave occurs at a point $P_{n}$ (referred to as the detach point), which is located at a distance $x_{n}$ along the trajectory of the bullet - see Fig. 7. When the listener is near the trajectory, the sound pulse (or sonic boom) is extremely loud. Similar to the muzzle blast wave, the shock wavefront travels away from its point of emission at the speed of sound. But, unlike the muzzle blast wavefront, it expands as a conical surface with the trajectory and nose of the bullet defining the axis and apex of the cone respectively. The angle $\theta_{n}$ (see Fig. 7) at the apex of the cone is referred to as the Mach angle, whose sine is equal to the reciprocal of the Mach number, which is defined as the ratio of the bullet's speed $V$ to the speed of sound $c^{23} \mathrm{~A}$ casual listener will hear the shock wave before the muzzle blast and instinctively look in the direction of its origin (the detach point) and confuse it with the actual direction of the firer, which coincides with the direction of the muzzle blast that arrives later - see Fig 7 .

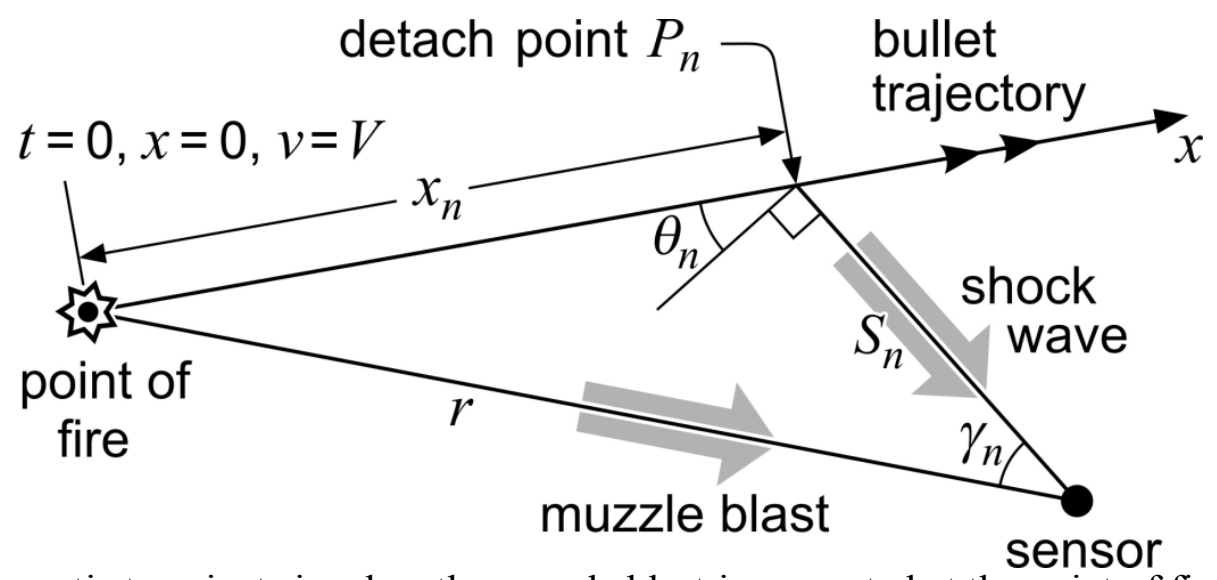

Figure 7. Acoustic transient signals - the muzzle blast is generated at the point of fire and the ballistic shock wave originates from the detach point along the bullet's trajectory. 


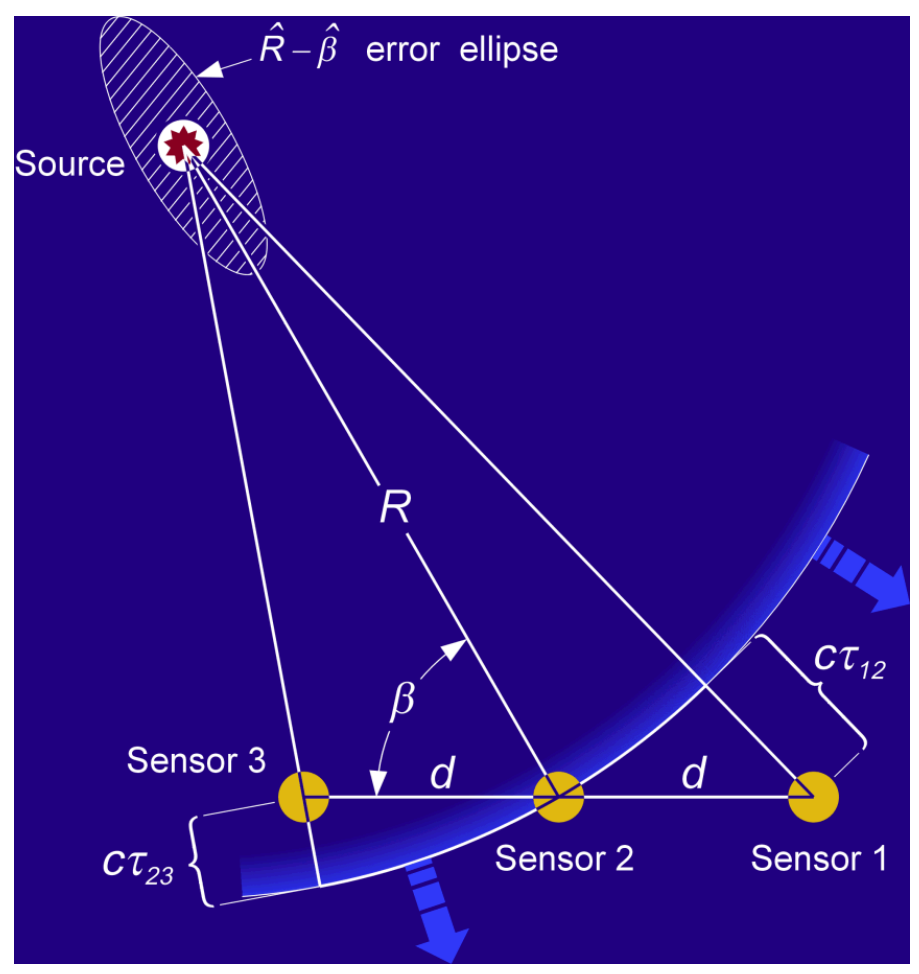

Figure 8. Source-sensor geometry for passive ranging by wavefront curvature.

By sensing these signals at spatially-separated sensors and applying various acoustical signal processing methods for sound source localization, it is possible to estimate the position of the firing point. One method, which relies only on the muzzle blast, is referred to as passive ranging by wavefront curvature ${ }^{24}$. The simplest sensor configuration for this method consists of three equally-spaced microphones positioned along a straight line - see Fig. 8. The basic principle is to estimate the wavefront's radius of curvature, which corresponds to the range of the firing point. Knowing the speed of sound travel in the atmosphere $(c)$ and the intersensor separation distance $(d)$, and measuring the differences in the arrival times $\left(\tau_{12}\right.$ and $\left.\tau_{23}\right)$ of the muzzle blast wavefront at adjacent sensor pairs, enables the calculation of the source range $R$ (from the middle sensor) and source bearing $\beta$ (with respect to the array axis) ${ }^{24}$. The results of applying this method to the passive ranging of real gunshot data from five different firing positions are shown in Fig. 9; typically 260 rounds were fired from each position. The variance of the source range estimates increases with range, while the bearing estimates for the serial conducted at the longest range $(475 \mathrm{~m})$ have a bias error which could be attributed to atmospheric refraction of the sound or uncertainty in the ground truth data of the firing position. The variances of both the range and bearing estimates can be reduced by increasing the effective sensor separation distance $(d \sin \beta)^{24}$. 

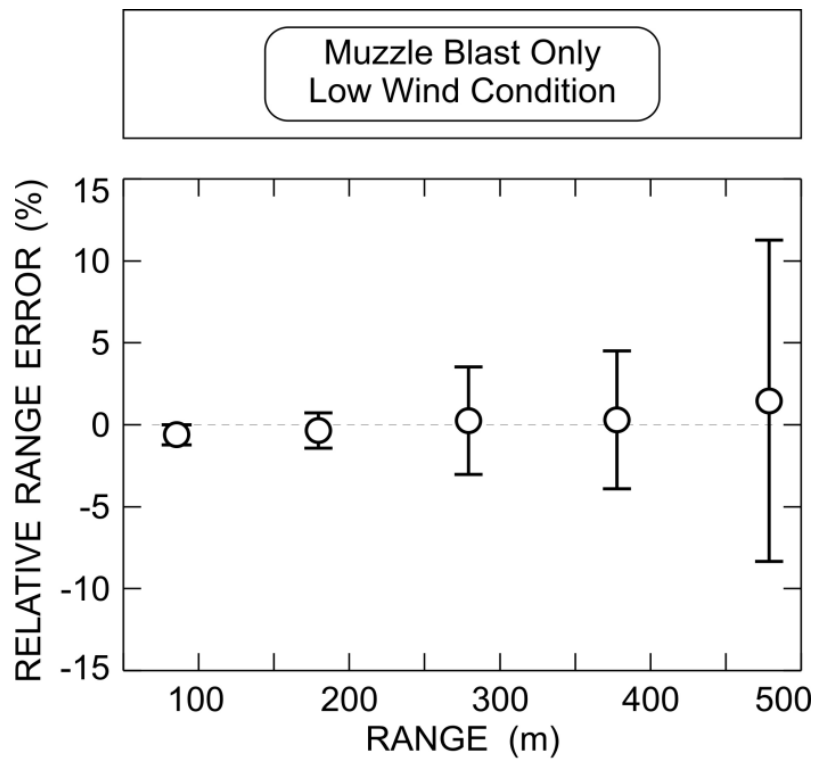

(a)

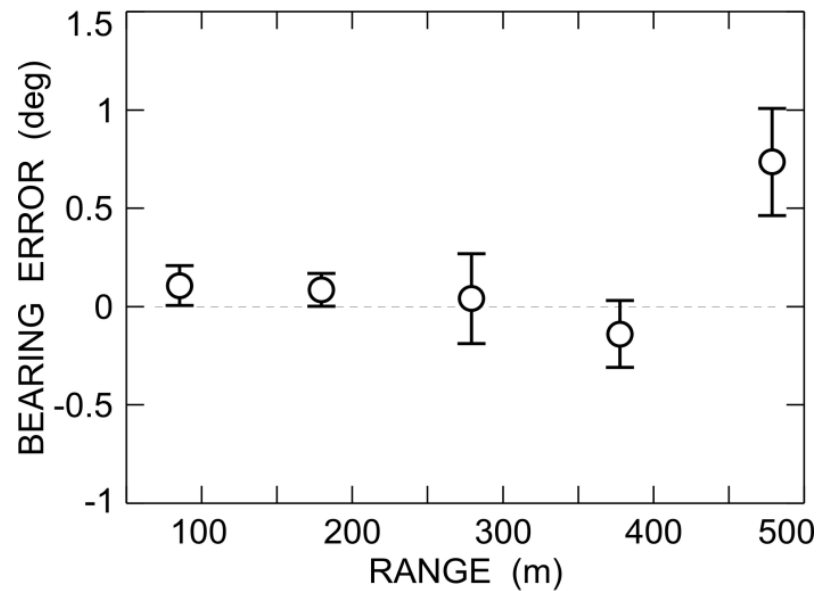

(b)

Figure 9. Variation with range of (a) relative range error and (b) bearing error for localizing the point of fire at five ranges using passive ranging by wavefront curvature of the muzzle blast wave only. 


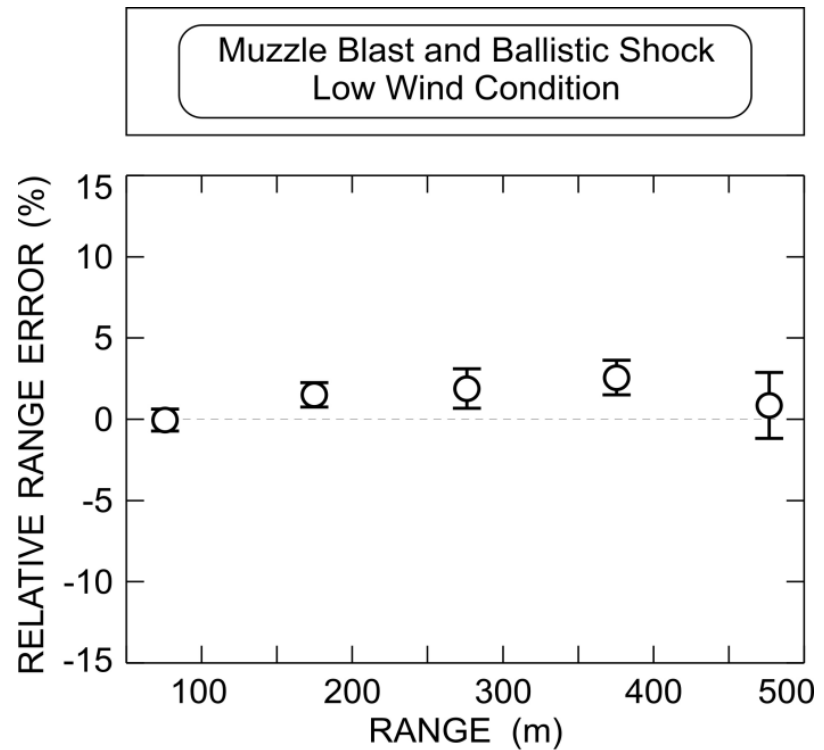

(a)

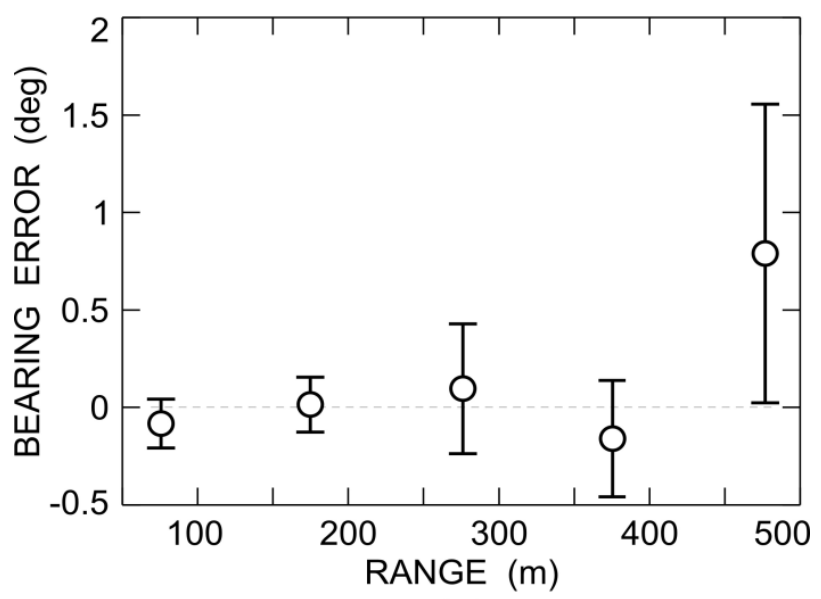

(b)

Figure 10. Similar to Figure 9 but for the ballistic model-based method which uses both the muzzle blast and shock wave information.

A second method, which uses both muzzle blast and shock wave information, is referred to as the ballistic model-based method for passive ranging of direct fire weapons ${ }^{25}$. Measuring the differences in the arrival times $\left(\Delta \tau_{n}=r / c-t\left(x_{n}\right)-s_{n} / c\right)$ and arrival angles $\gamma_{n}$ of the muzzle blast and shock waves at a sensor node $n$ (a small baseline sensor array) enables the range $r$ to be estimated - see Fig. 7. For a bullet travelling with a constant velocity $(V)$, $r=c \Delta \tau_{n} /\left(1-\cos \gamma_{n}\right)^{25}$. In practice, range estimates based on the constant bullet velocity assumption can have significant errors (especially at long source ranges), necessitating the development of a ballistic model-based approach that accounts for the deceleration of the bullet along its trajectory. The shock wavefront is better represented (visualized) as a half prolate spheroid (pointed oval shape like an American football) for a decelerating bullet, rather than as a conical surface for a bullet travelling with a constant velocity. The ballistic parameters, which 
must be known a priori or estimated in situ, are the bullet's initial velocity and the ballistic constant (which depends on the bullet's mass, cross-sectional area and aerodynamic drag). The results of applying this method to the passive ranging of real gunshot data from the five different firing positions are shown in Fig. 10. When compared with the passive ranging by wavefront curvature method (Fig. 9), the variances of the ranges of the firing positions are reduced when estimated using the ballistic model-based method, most notably at the longer source ranges. The converse is true for the bearings of the source positions because of the shorter baseline of the sensor array used with the ballistic model-based method. Additionally, when the source ranges are estimated using the conventional method which assumes a constant bullet velocity, they are found to have significant bias errors especially at the longer firing ranges. Also, the radial error, which is defined as the separation distance between the estimated and actual firing positions, is found to be dependent on the caliber of the bullet - the conventional method's radial errors are much larger for $5.56 \mathrm{~mm}$ rounds than for $7.62 \mathrm{~mm}$ caliber ammunition.

Currently under development is a third localization method ${ }^{26}$ that relies only on the ballistic shock wave information, which is advantageous when the received muzzle blast is absent due to the use of a sound suppressor (silencer) or weak due to the high transmission loss (spreading loss plus absorption loss) suffered by the acoustic signal when its propagation path from source to sensor is long. Another advantage of this method occurs when there is simultaneous fire from different firing positions as each shock wave signal is not required to be associated with a corresponding muzzle blast signal as is the case with the ballistic model-based method. A new method proposed by the authors is simultaneous localization and classification, which uses both the muzzle blast and shock wave information received by a next-generation network of spatially-distributed unattended ground sensors comprising "low-cost sensor nodes operating on shoestring power budgets for years at a time in potentially hostile environments without hope of human intervention".

\section{Summary}

In this article we have presented three examples of signal processing approaches in physical and engineering acoustics. In time reversal, we exploit a principle in physical acoustics to enhance the detection of flaws in plates and structures. In gearbox monitoring we show how sophisticated statistical techniques such as principle component analysis and blind source separation can be employed to solve a difficult and important problem in structural and machine monitoring. Finally, in point of fire localization we see how both wavefront curvature and shock front propagation can be combined to improve estimates of the origin of a bullet fired from a gun. We hope this gives a flavor of the variety of approaches and applications of signal processing to physical and engineering acoustics.

\section{Acknowledgements}

Lawrence Livermore National Laboratory is operated by Lawrence Livermore National Security, LLC, for the U.S. Department of Energy, National Nuclear Security Administration under Contract DE-AC52-07NA27344. 


\section{References}

${ }^{1}$ For more information contact author B.E.A. Electronic mail: bea@byu.edu

${ }^{2}$ M. Fink, “Time reversed acoustics," Physics Today 50, $34-40$ (1997).

${ }^{3}$ B. E. Anderson, M. Griffa, C. Larmat, T. J. Ulrich, and P. A. Johnson, “Time reversal," Acoustics Today 4(1), 5-16 (2008).

${ }^{4}$ A. Parvulescu and C. S. Clay, "Reproducibility of signal transmission in the ocean," Radio and Electrical Engineering 29, 223-228 (1965).

${ }^{5}$ K. G. Sabra, P. Roux, H.-C. Song, W. S. Hodgkiss, W. A. Kuperman, T. Akal, and J. M. Stevenson, "Experimental demonstration of iterative time-reversed reverberation focusing in a rough waveguide. Application to target detection," Journal of the Acoustical Society of America 120(3), 1305-1314 (2006).

${ }^{6}$ H.-C. Song, J. S. Kim, W. S. Hodgkiss, W. A. Kuperman, and M. Stevenson, "High-rate multiuser communications in shallow water," Journal of the Acoustical Society of America 128(5), 2920-2925 (2010).

${ }^{7}$ H.-C. Song, W. S. Hodgkiss, and P. A. van Walree, "Phase-coherent communications without explicit phase tracking," Journal of the Acoustical Society of America 128(3), 969-972 (2010).

${ }^{8}$ J.L. Robert and M. Fink, "Green's function estimation in speckle using the decomposition of the time reversal operator: Application to aberration correction in medical imaging," Journal of the Acoustical Society of America 123(2), 866-877 (2008).

${ }^{9}$ S. Dos Santos and Z. Prevorovsky, "Imaging of human tooth using ultrasound based chirpcoded nonlinear time reversal acoustics," Ultrasonics 51(6), 667-674 (2011).

${ }^{10}$ B. E. Anderson, M. Griffa, T. J. Ulrich, P.-Y. Le Bas, R. A. Guyer, and P. A. Johnson, "Crack localization and characterization in solid media using time reversal techniques," American Rock Mechanics Association, \#10-154 (2010).

${ }^{11}$ B. E. Anderson, M. Griffa, P.-Y. Le Bas, T. J. Ulrich, and P. A. Johnson, "Experimental implementation of reverse time migration for nondestructive evaluation applications," Journal of the Acoustical Society of America Express Letters 129(1), EL8-EL14 (2011).

${ }^{12}$ C. Larmat, R. A. Guyer, and P. A. Johnson, "Tremor source location using time reversal: Selecting the appropriate imaging field," Geophysical Research Letters 36, L22304 (2009).

${ }^{13}$ C. Larmat, R. A. Guyer, and P. A. Johnson, "Time reversal methods in seismology," Physics Today 63(8), 31-35 (2010). 
14 T. J. Ulrich, M. Griffa, and B. E. Anderson, "Symmetry-based imaging condition in time reversed acoustics," Journal of Applied Physics 104(6), 064912 (2008).

${ }^{15}$ B. E. Anderson, M. Griffa, L. Huang, P. A. Johnson, P.-Y. Le Bas, and T. J. Ulrich, “A comparison of time reversal imaging techniques of two dimensional samples," XIII International Conference on Nonlinear Elasticity and Materials, Aix en Provence, France, June (2008).

${ }^{16}$ For more information contact author M.J.R.

17 M. Lebold et al. 2000 Proceedings of the 54th Meeting of the Society for Machinery Failure Prevention Technology, Virginia Beach, VA, 623-634. Review of vibration analysis methods for gearbox diagnostics and prognostics.

18 J. E. Nicks and G. Krishnappa 1995 IMechE Conference Transactions: 2nd International Conference on Gearbox Noise, Vibration and Diagnostics, May1995, 81-89.

${ }^{19}$ W. J. Wang and P. D. McFadden 1993 Mechanical Systems and Signal Processing 7, 193-203. Early detection of gear failure by vibration analysis.

20 A. J.Miller 1999 M.S. thesis, The Graduate School of The Pennsylvania State University. A new wavelet basis for the decomposition of gear motion error signals and its application to gearbox diagnostics.

${ }^{21}$ M. J. Roan and J. Erling, and L.H. Sibul, "A New, Non-Linear, Blind Source Separation Algorithm for Gear Tooth Failure Analysis," The Journal of Mechanical Systems and Signal Processing, Vol. 16, No. 5,pp. 719-740, September, 2002.

${ }^{22}$ For more information contact authors B.G.F. and K.W.L.

${ }^{23}$ A. D. Pierce, Acoustics - An Introduction to Its Physical Principles and Applications, pp. 606615, Acoustical Society of America, New York, 1994.

${ }^{24}$ B. G. Ferguson, "Variability in the passive ranging of acoustic sources in air using a wavefront curvature technique", Journal of the Acoustical Society of America, Vol. 108, No. 4, pp. 15351544, October 2000.

${ }^{25}$ K. W. Lo and B. G. Ferguson, "A ballistic model-based method for ranging direct fire weapons using the acoustic muzzle blast and shock wave", Proceedings of the 2008 Intelligent Sensors, Sensor Networks and Information Processing Conference, CD ISBN: 978-1-4244-29578 pp. 453-458, December 2008.

${ }^{26}$ K. W. Lo and B. G. Ferguson, "Acoustic methods for localizing small arms fire”, Submitted for publication to the Journal of the Acoustical Society of America, July 2011. 


\section{Figure Captions}

Figure 1. Illustration of the reverse time migration process in a free space with a scatterer at location $C$. The propagation times for each path are included for the reader's reference. (a) Forward propagation step. (b) Backward propagation step.

Figure 2. (a) Photograph of a semicircular aluminum plate which has the letters "LANL" milled out of it and steel letters "EES-11" glued onto it. S and R denote the source and receiver transducers named according to the forward propagation usage. (b) RTM image of the other side of this plate (mirror image is displayed). Amplitude is in $\mathrm{dB}$ units with an arbitrary reference.

Figure 3. Simple spur gear and pinion setup

Figure 4. Crack formation and growth at the base of a gear tooth

Figure 5. High Level Diagram of the Blind Source Separation Algorithm

Figure 6. Result of Blind Source Separation Processing for a healthy gear (top) and for a gear that is beginning to break (bottom)

Figure 7. Acoustic transient signals - the muzzle blast is generated at the point of fire and the ballistic shock wave originates from the detach point along the bullet's trajectory.

Figure 8. Source-sensor geometry for passive ranging by wavefront curvature.

Figure 9. Variation with range of (a) relative range error and (b) bearing error for localizing the point of fire at five ranges using passive ranging by wavefront curvature of the muzzle blast wave only.

Figure 10. Similar to Figure 9 but for the ballistic model-based method which uses both the muzzle blast and shock wave information. 